\title{
Subjective Evaluation of Directional Properties of Loudspeaker Systems with the Analog and Digital Crossover Networks
}

\author{
M. Niewiarowicz, T. Galiński \\ Department of Otolaryngology, Poznań University of Medical Sciences, Przybyszewskiego 49, 60-355 Poznań, Poland \\ Institute of Acoustics, Faculty of Physics, Adam Mickiewicz University, Umultowska 85, 61-614 Poznań, Poland
}

\begin{abstract}
The aim of this study was to determine the way in which loudspeaker systems with the analog and digital crossover networks were subjectively evaluated. The object of the study were two loudspeaker systems, differing in both the position of speakers on the front panel of enclosure and configuration of the crossover networks: 2.5-way and 3-way systems with the slope of frequency response of $12 \mathrm{~dB} /$ octave in the cut-off regions. The investigations were conducted for 5 angles from the front hemisphere in the horizontal plane. Test signals were fragments of music and speech. The recordings of these signals were conducted in an anechoic chamber using an artificial head and then presented to the listeners through headphones. Five normally-hearing listeners aged 18-50 years took part in the experiment. Listening tests were carried out in two stages. In the first stage listeners were listening to test signals grouped in triads and their task was to identify pairs of signals the most and the least similar to each other. In the second stage a parametric evaluation was performed. It consisted in assigning five numbers, corresponding to the intensity of the attribute of perception space (evaluated on a scale of $0-10$ ) to each of test signals. The attributes of perception space included: sharpness, clearness, fullness, lack of distortions and loudness. Using the method of multidimensional scaling and correlation analysis it was possible to relate a particular dimension of the multidimensional space to the attributes of the perception space. It was found that the intrinsic attributes responsible for the subjective evaluation of loudspeaker systems with the analog and digital crossover network were sharpness, clearness and loudness.
\end{abstract}

DOI: 10.12693/APhysPolA.123.974

PACS: 43.38. $+\mathrm{n}, 43.66 .+\mathrm{y}$

\section{Introduction}

The problem of directional properties of electroacoustic transducers acquire a special significance in the case of loudspeaker systems used in the process of stereophonic transmission or in the process of sound reinforcement. The investigations carried out on loudspeaker systems revealed some essential differences in the course of the frequency response on the system axis and outside it, particularly for the frequencies from cut-off region of crossover networks. The subjective evaluation of the loudspeaker system may depend, therefore, on the spatial conditions in which it has been performed. Directivity of sound source plays thus an important role in the process of sound reinforcement and the knowledge of directional properties of sound source is of essential importance for appropriate modeling of physical phenomena which accompany this process [1].

The quality of the sounds reproduced by the loudspeaker systems depends significantly on the transmission of transients. This becomes all the more obvious when one realizes that the real sounds (e.g. speech, music, environmental sounds) are signals of purely transient nature. The distortions occurring in transients depend on both the width of transmission range and irregularity of frequency characteristic. The phenomena mentioned above influence to a great degree the directivity of radiation of loudspeaker systems. The investigations carried out, among others, by one of the present authors showed, that directional properties of loudspeaker systems in steady and transient states differ significantly $[2-4]$.

Application of the digital signal processing (DSP) technique in construction of the crossover network of loudspeaker systems enables to create their transmission properties, and hence also directional characteristics, to a much greater degree than in the analog systems [5]. Changes in the cut-off frequency and the slope of the amplitude characteristic of the crossover filters can bring about significant differences in the directional characteristics of the loudspeaker systems and thus differences in the resulting effect of sound reinforcement in a room. The main demand as to the directional characteristics of a loudspeaker system is to ensure the uniform sound propagation into the front hemisphere. The efficiency of sound propagation into the front hemisphere is ensured by a set of digital filters of FIR (finite impulse response) class $[6,7]$ ensuring linear phase distortions, which cannot be provided either by active or passive Butterworth or Czebyszew filters. The great advantage of these systems consists in possibility of changing, in a simple way, the parameters of crossover network which would cause the modification of the frequency and/or directional characteristics. But in fact, the most important criterion of selection of suitable system should be connected with the subjective evaluation of perceived acoustical sensations $[8-10]$. The main purpose of this study was to de- 
termine how the location of the listener relative to the loudspeaker system affects its subjective evaluation and which attributes of perception space are responsible for this. The special emphasis was placed on examination of the influence of the conversion of an analog to digital crossover network.

\section{Experimental investigations}

Experimental investigations were realized in two stages. The first stage consisted in selecting loudspeaker systems and signals, calibration of measurement system and headphones and finally, in registration of test signals for the subjective evaluation. In the second stage subjective evaluations were conducted according to the procedure prepared.

\subsection{Objective investigations \\ 2.1.1. Object of study}

The object of study was two loudspeaker systems that differed in the number of loudspeakers and their arrangement on the front panel of enclosure. However, the most important difference was related to the use of different types of crossover networks - 2.5-way and 3-way crossover networks with the analog and digital filters:

- loudspeaker system LS-I - a 2.5-way system with the following cut-off frequencies:

- $1000 \mathrm{~Hz}$ - low pass range

- $1800 \mathrm{~Hz}$ - low-mid pass range

- $3000 \mathrm{~Hz}$ - high pass range

for both the analog and digital filters.

- loudspeaker system LS-II - 3-way system with the following cut-off frequencies:

- $1500 \mathrm{~Hz}$ - low pass range

- $1500-6500 \mathrm{~Hz}$ - mid-band-pass range

- $6500 \mathrm{~Hz}$ - high pass range

for the analog filter. The mid range digital filter was selected by the DSP technique in such a way that the resultant frequency characteristic of the whole crossover network was as flat as possible.

In all cases the slope of the frequency characteristic in the cut-off regions was $12 \mathrm{~dB} /$ octave. The frequency characteristics for the analog and digital crossover networks are shown in Fig. 1.

As it was mentioned in the Introduction, the quality of the sounds reproduced by the loudspeaker systems depends significantly on transmission of transients. Transients occur most frequently for frequencies lying in the cut-off regions of the crossover filters and influence the pattern of directional characteristic of loudspeaker system. The exemplary graphs for both loudspeaker systems are presented in Figs. 2 and 3. The measurements were conducted, according to the procedure described in [4], for the $1 / 3$ oct. band noise excitation signals with center frequencies of $f_{\mathrm{c}}=1900 \mathrm{~Hz}$ and $f_{\mathrm{c}}=1400 \mathrm{~Hz}$ for LS-I and LS-II, respectively.

As can be seen from the above figures, the directional characteristics for loudspeaker systems with the analog
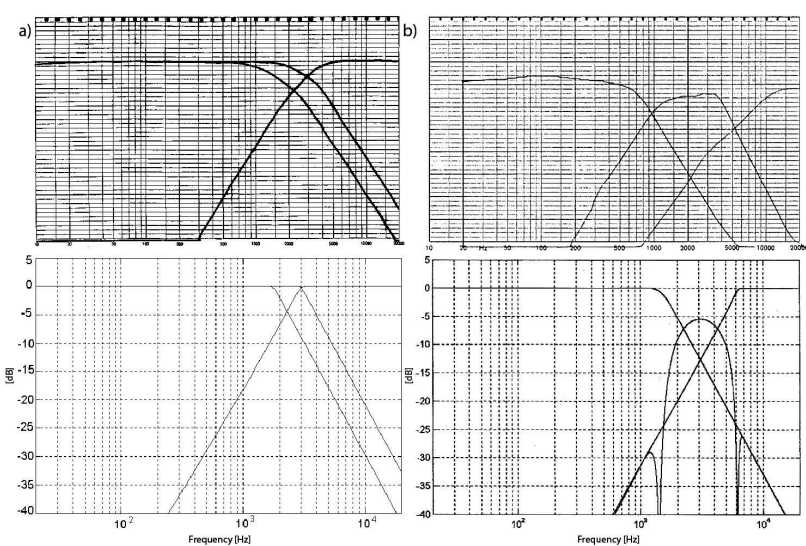

Fig. 1. Frequency characteristics of the crossover networks: (a) LS-I; (b) LS-II; analog (upper diagram) and digital (bottom diagram).
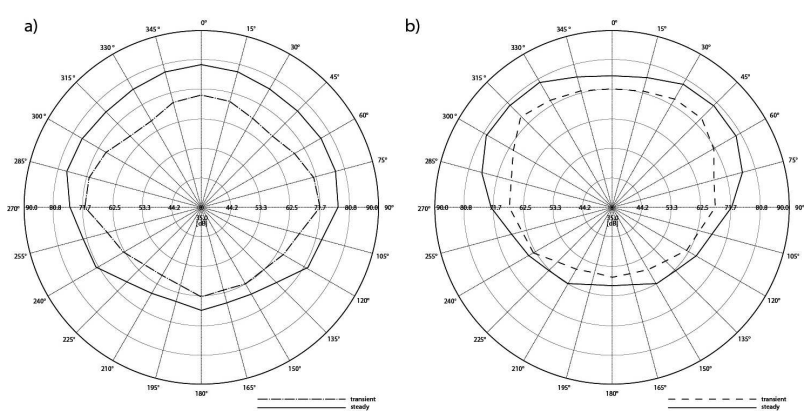

Fig. 2. Directional characteristics of the loudspeaker system LS-I for the initial transient and steady state with the analog (a) and digital (b) crossover network for $f_{\mathrm{c}}=1900 \mathrm{~Hz}$

(LS-I,II a) and digital (LS-I,II b) crossover networks differ. Moreover, the differences are also evident, independently for each loudspeaker system, if we compare the directional characteristics of the steady and transient states. It is therefore assumed that these differences will also affect the subjective evaluation for different angles of observation.
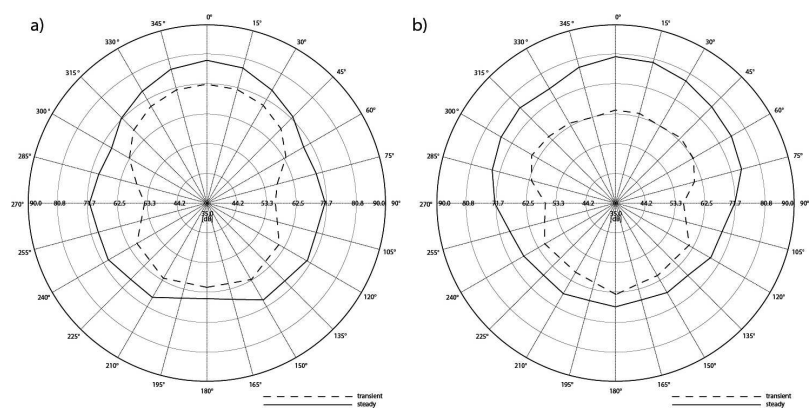

Fig. 3. Directional characteristics of the loudspeaker system LS-II for the initial transient and steady state with the analog (a) and digital (b) crossover network for $f_{\mathrm{c}}=1400 \mathrm{~Hz}$. 


\subsubsection{Registration of test signals}

Test signals were selected from "Denon Professional Test CDs". These signals were fragments of music and speech, however the choice was based on the following criteria:

- music -signal should be balanced, i.e. has uniform spectral energy distribution, without emphasizing bass and treble

- speech - listener should not focus on understanding the text, but on the acoustic features of the signal.

Finally, as test signals, chamber music and Japanese speech were used. Waveforms of selected signals are shown in Fig. 4.
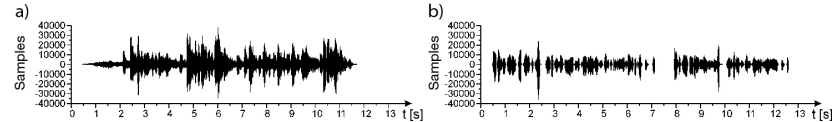

Fig. 4. Waveforms of test signals: (a) chamber music and (b) Japanese speech.

The basic assumption to perform subjective tests was that they will be carried out using headphones and therefore, in the first step their registration should be made. The registration of test signals was performed for five angles in the horizontal plane: $0^{\circ}, 15^{\circ}, 30^{\circ}, 45^{\circ}$ and $60^{\circ}$ relative to the symmetry axis of loudspeaker system using artificial head (Neumann) placed at the distance of $3 \mathrm{~m}$ and computer $\mathrm{PC}$ which also controlled rotation of a turntable.

Prior to registration, calibration of measurement setup was performed. The loudspeaker system was placed on the turntable at the angle of $0^{\circ}$ and artificial head was replaced by the measuring microphone. Calibration signal was white noise. For each loudspeaker system under investigation the gain was adjusted so that the measured value was equal to $80 \mathrm{~dB}$ SPL. The investigations were conducted in an anechoic chamber.

\subsection{Subjective experiments}

Since the main aim of this study was to determine the effect of conversion of the analog to digital crossover network, listening tests were prepared separately for loudspeaker system LS-I and loudspeaker system LS-II. For each signal a name that identifies both the type of filter (analog or digital) and registration angle was assigned. For the loudspeaker systems with the analog filter $A_{n}$ and digital filter $D_{n}$ where $n$ denotes numbers of successive angles for which the registration of test signals was made (see 2.2.1). The listening tests were conducted in a listening room using headphones (Sennheiser). In both experiments, 5 listeners aged 18-50 years took part. Listeners were selected on the basis of the correct results of tonal audiometry i.e. their auditory thresholds were lower than $10 \mathrm{~dB}$ HL (hearing level).

\subsubsection{Overall evaluation}

The overall evaluation was conducted by means of the of triadic comparison method [11]. The subject's task was to determine a pair of the most similar sounds and a pair of the least similar sounds in every triad (see Note). The indicated pair of signals was assigned a certain weight:

- 2 - a pair of the least similar sounds

- 0 - a pair of the most similar sounds

- 1 - other pair.

Note: This method requires grouping all test signals into triads (all combinations of 3 signals from the total number of signals $N$ ). The number of these combinations (triads) defines Newton's binomial. In the described studies $N=10$ (2 acoustic signals and 5 angles) and therefore the number of triads for one loudspeaker system is equal

$$
\left(\begin{array}{c}
N \\
3
\end{array}\right)=\frac{N !}{3 !(N-3) !}=\frac{10 !}{3 ! 7 !}=135 .
$$

From the point of view of accuracy of the experiment, each listening test should therefore contain the total number of 135 triads and should be performed in a single listening session. This would imply a significant increase in the duration of the session and thus, significant deterioration of subjects' perception abilities. For this reason, it was necessary to limit the number of triads used in the experiment. This was possible only by reducing the number of angles for which the tests were to be carried out. This problem was solved by performing 4 listening sessions (135 triads for each session) for LS-I and LS-II with both the analog and digital crossover networks. As a result of conducting the variance analysis (ANOVA) it was stated that insignificant differences in the evaluation $(p>0.05)$ were appearing for signals corresponding to angles $0^{\circ}$ and $15^{\circ}$. Therefore, the overall evaluation was performed for test signals corresponding to the angles $0^{\circ}$, $30^{\circ}, 45^{\circ}$ and $60^{\circ}$. In this way, the number of triads in one test decreased to 56 ( $N=8$ in the above equation) and numbering has been adopted the following form:

analog $A_{1} \quad A_{2} \quad A_{3} \quad A_{4}$

$$
\text { - } \quad 0^{\circ} 30^{\circ} 45^{\circ} 60^{\circ}
$$

digital $D_{1} D_{2} D_{3} D_{4}$.

Listening test was controlled by a computer program. Its main task was to select the successive triads containing signals that differed significantly from each other.

\subsubsection{Parametric evaluation}

The parametric evaluation was carried out by means of the rating scale method. The subjects' task was to assess each sound on a 0-10 scale. These scales refer to the attributes of perception space: sharpness, clearness, fullness, lack of distortions and loudness [12, 13]. Value equal to 0 on the scale indicated a complete lack of sensation connected with given attribute while the value of 10 indicated the maximum sensation. During the listening test the subjects could refer anytime to a definition list of attributes. The attributes have been defined as follows: - sharpness - the sound contains components whose mid- and high-frequency levels are to high 
TABLE I

Ranking of test signals in two-dimensional perception space for loudspeaker systems LS-I and LS-II with analog and digital crossover networks for music and speech.

\begin{tabular}{|c|c|c|c|c|c|c|c|c|c|c|c|c|c|c|c|c|}
\hline & \multicolumn{8}{|c|}{ music } & \multicolumn{8}{|c|}{ speech } \\
\hline & \multicolumn{4}{|c|}{ Dimension I } & \multicolumn{4}{|c|}{ Dimension II } & \multicolumn{4}{|c|}{ Dimension I } & \multicolumn{4}{|c|}{ Dimension II } \\
\hline \multirow{3}{*}{ LS-I } & $A_{4}$ & $A_{3}$ & $A_{2}$ & $A_{1}$ & $A_{4}$ & $A_{1}$ & $A_{2}$ & $A_{3}$ & $A_{4}$ & $A_{3}$ & $A_{2}$ & $A_{1}$ & $A_{3}$ & $A_{4}$ & $A_{2}$ & $A_{1}$ \\
\hline & $D_{4}$ & $D_{3}$ & $D_{2}$ & $D_{1}$ & $D_{3}$ & $D_{2}$ & $D_{1}$ & $D_{4}$ & $D_{4}$ & $D_{3}$ & $D_{2}$ & $D_{1}$ & $D_{2}$ & $D_{1}$ & $D_{4}$ & $D_{3}$ \\
\hline & ${ }^{A_{1}}{ }_{A_{2}}$ & ${ }^{A_{3}}{ }_{A_{4}}$ & $\begin{array}{l}D_{1} \\
\quad D_{2}\end{array}$ & $\begin{array}{l}D_{3} \\
D_{4}\end{array}$ & $\stackrel{A_{1}}{D_{1}}$ & $\stackrel{D_{2}}{A_{2}}$ & $\begin{array}{c}D_{3} \\
A_{3}\end{array}$ & $\stackrel{A_{4}}{D_{4}}$ & ${ }^{A_{2}}{ }_{A_{1}}$ & ${ }^{A_{3}}{ }_{A_{4}}$ & $\mid \begin{array}{l}D_{1} \\
\quad D_{2}\end{array}$ & $\begin{array}{c}D_{3} \\
\quad D_{4}\end{array}$ & $\stackrel{D_{1}}{A_{1}}$ & $\begin{array}{l}D_{2} \\
A_{2}\end{array}$ & ${ }_{A_{3}}$ & ${ }^{D_{4}}{ }_{A_{4}}$ \\
\hline \multirow{3}{*}{ LS-II } & $A_{4}$ & $A_{3}$ & $A_{2}$ & $A_{1}$ & $A_{4}$ & $A_{1}$ & $A_{2}$ & $A_{3}$ & $A_{4}$ & $A_{3}$ & $A_{2}$ & $A_{1}$ & $A_{4}$ & $A_{1}$ & $A_{2}$ & $A_{3}$ \\
\hline & $D_{4}$ & $D_{3}$ & $D_{2}$ & $D_{1}$ & $D_{4}$ & $D_{2}$ & $D_{3}$ & $D_{1}$ & $D_{4}$ & $D_{3}$ & $D_{2}$ & $D_{1}$ & $D_{4}$ & $D_{2}$ & $D_{3}$ & $D_{1}$ \\
\hline & $A_{2}$ & $A_{1}$ & $D_{4}$ & $D_{3}$ & $D_{4}$ & $D_{3}$ & $D_{2}$ & $D_{1}$ & $A_{1}$ & $A_{3}$ & $D_{1}$ & $D_{3}$ & $D_{1}$ & $A_{2}$ & $A_{3}$ & $D_{4}$ \\
\hline
\end{tabular}

- clearness - the sound is pure, clear; different instruments and voices can be easily distinguished, onsets and transients in the music can be easily perceived

- fullness - the sound contains the entire spectrum without any limitations, at least in the bass range

- lack of distortions - indicates a pure sound, without distortions, one which is not harsh, hiss or rumbling.

Each listener performed (during one listening session) the evaluation of individual attributes of perception space for all test signals. Every session was repeated five times on different days.

\section{Results of subjective investigations}

The results of the overall evaluation (dissimilarities between sounds) have been analyzed by the method of multidimensional scaling of individual differences (INDSCAL) [14]. The calculations revealed that two dimensions was the optimal number of dimensions of metric space, enabling to describe the configuration of experimental data. According to these calculations a set of arrengements of test signals in two-dimensional perception space was obtained for each loudspeaker system. The complete set of arrangements is presented in Table I.

The rankings in Table I refer to two cases:

- when the signals of the same type i.e. $A_{n}$ or $D_{n}$ are taken into account - in this case the ranking reflects the differences in the perception of signals depending on the angle of observation

- when all signals $A_{n}$ and $D_{n}$ are considered together - in this case the ranking reflects the differences in perception of signals depending on the type of crossover network.

As can be seen from the data in Table I the ranking 4, 3, 2, 1 for dimension I is repeated for both loudspeaker systems, regardless of whether the analog or digital crossover networks were used. For dimension II no uniform rankings are observed.

The next stage of the research was aimed to finding a relationship between the results obtained in both the overall evaluation and the parametric evaluation. To assign a particular attribute of the perception space to one of the dimensions of dissimilarity space, the correlation analysis was performed. To calculate the correlation coefficients the Pearson linear correlation was used. The obtained coefficients are summarized in Table II.

Based on the results received it was possible to assign certain attributes of the perception space to different dimensions of dissimilarity space. As a fitting criterion, the value of the correlation coefficient $R>0.75$ was adopted. It can be seen easily that the correlation coefficients for the Dimension II, do not meet the required criterion even in one case. Thus, in all cases, the attributes of perception space can only be assigned to the ranking in Dimension I. As expected, the most frequently repeated ranking has been assigned to a single parameter, namely the sharpness. The following figures show the distribution of points corresponding to each test signals in twodimensional space, and the attributes of perception space correlated with them.

Since the sharpness determines the evaluation connected with angular resolution, the calculation was carried out to designate the so-called minimum perceived change in position, defined as the smallest difference between the signals are perceived by the listener. For this purpose, based on the results of the parametric evaluation, the mean values (for all listeners) for individual signals as well as the standard deviation were calculated.

TABLE III

Values of the minimum perceived change in position

\begin{tabular}{l|c|c|c|c}
\hline \hline & \multicolumn{2}{|c|}{ LS-1 } & \multicolumn{2}{c}{ LS-2 } \\
\cline { 2 - 5 } & music & speech & music & speech \\
\hline $30^{\circ}-45^{\circ}$ & $0^{\circ}-30^{\circ}$ & $30^{\circ}-60^{\circ}$ & $0^{\circ}-30^{\circ}$ & \\
analog & & $30^{\circ}-45^{\circ}$ & & $45^{\circ}-60^{\circ}$ \\
& & $45^{\circ}-60^{\circ}$ & & \\
\hline \multirow{3}{*}{ digital } & $30^{\circ}-45^{\circ}$ & $0^{\circ}-30^{\circ}$ & $30^{\circ}-45^{\circ}$ & $0^{\circ}-30^{\circ}$ \\
& $45^{\circ}-60^{\circ}$ & & & $30^{\circ}-45^{\circ}$ \\
& & & & $45^{\circ}-60^{\circ}$
\end{tabular}


TABLE II

Correlation coefficients for results obtained in the overall and parametric evaluation.

\begin{tabular}{|c|c|c|c|c|c|c|c|c|}
\hline & \multicolumn{4}{|c|}{ Dimension I } & \multicolumn{4}{|c|}{ Dimension II } \\
\hline & \multicolumn{2}{|c|}{ LS-1 } & \multicolumn{2}{|c|}{ LS-2 } & \multicolumn{2}{|c|}{ LS-1 } & \multicolumn{2}{|c|}{ LS-2 } \\
\hline & music & speech & music & speech & music & speech & music & speech \\
\hline $\begin{array}{ll}\text { a } & \text { sharpness }\end{array}$ & 0.97 & 0.98 & 0.97 & 0.91 & 0.44 & -0.50 & 0.04 & 0.23 \\
\hline $\mathrm{n} \quad$ fullness & 0.48 & 0.93 & 0.87 & 0.72 & 0.21 & -0.53 & 0.16 & 0.28 \\
\hline a clearness & 0.88 & 0.72 & 0.41 & 0.80 & 0.10 & -0.42 & 0.43 & 0.24 \\
\hline 1 lack of $\mathrm{d}$. & 0.64 & 0.07 & 0.45 & 0.28 & -0.13 & -0.10 & -0.28 & -0.27 \\
\hline $\begin{array}{ll}\text { o } & \text { loudness } \\
\mathrm{g} & \\
\end{array}$ & 0.94 & 0.86 & 0.88 & 0.80 & -0.01 & -0.40 & -0.10 & 0.20 \\
\hline d i & & & & & & & & \\
\hline i shal & 0.94 & 0.86 & 0.98 & 0.97 & .39 & -0.46 & -0.10 & -0.38 \\
\hline $\mathrm{g} \quad$ fullness & 0.79 & 0.85 & 0.70 & 0.68 & 0.16 & -0.37 & -0.29 & -0.04 \\
\hline i clear & 0.35 & 0.56 & -0.35 & 0.36 & 0.01 & -0.11 & 0.08 & 0.13 \\
\hline $\mathrm{t}$ lack of $\mathrm{d}$. & 0.58 & -0.08 & 0.04 & -0.08 & -0.39 & -0.31 & 0.07 & 0.24 \\
\hline $\begin{array}{l}\text { a loudness } \\
\text { a d }\end{array}$ & 0.83 & 0.75 & 0.76 & 0.80 & 0.48 & -0.50 & -0.20 & -0.34 \\
\hline $\mathrm{n}$ i sharpness & 0.62 & 0.65 & 0.06 & 0.67 & -0.28 & -0.16 & 0.58 & 0.18 \\
\hline a $g$ fulln & 0.57 & 0.73 & 0.36 & 0.52 & 0.39 & -0.53 & 0.16 & 0.28 \\
\hline 1 i clearness & 0.77 & 0.75 & 0.76 & 0.74 & 0.03 & 0.13 & 0.03 & 0.01 \\
\hline o t lack & 0.61 & 0.25 & 0.71 & 0.03 & 0.23 & -0.01 & -0.28 & -0.09 \\
\hline $\mathrm{g}$ a loudness & 0.66 & 0.79 & 0.28 & 0.75 & -0.39 & 0.05 & 0.66 & 0.20 \\
\hline
\end{tabular}

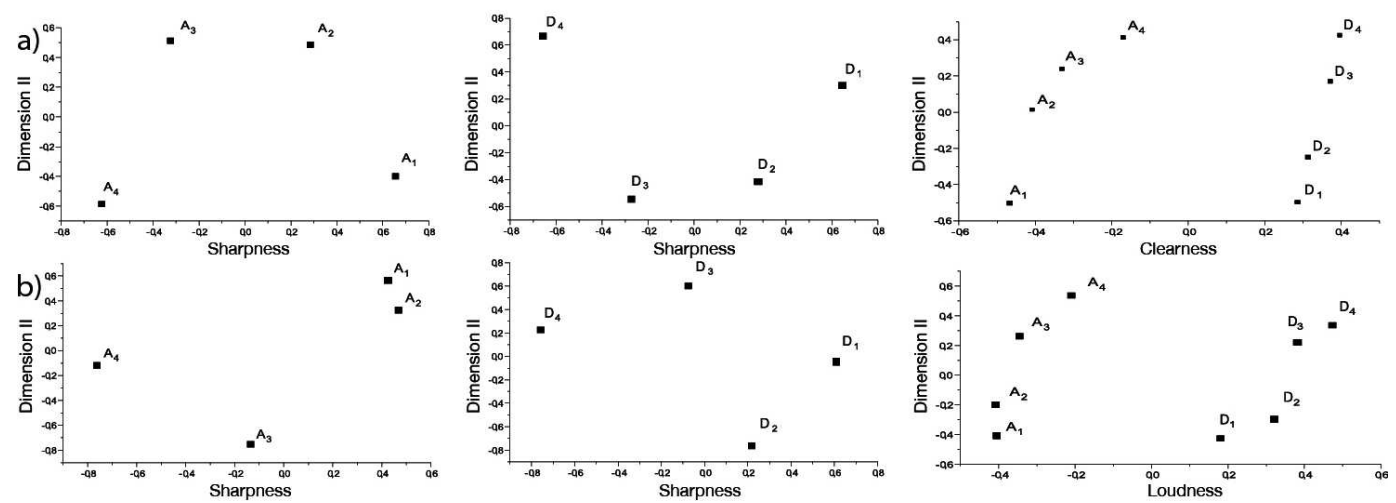

Fig. 5. Attributes of the perception space correlated with the dissimilatory space for loudspeaker system LS-I: (a) music and (b) speech.
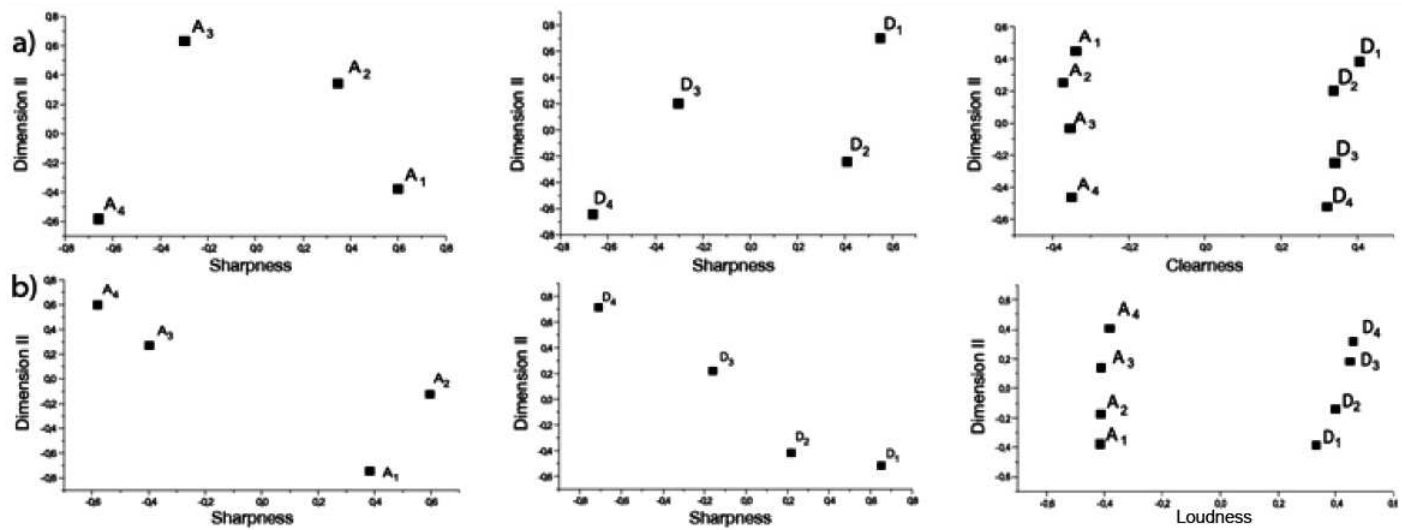

Fig. 6. Attributes of the perception space correlated with the dissimilatory space for loudspeaker system LS-II: (a) music and (b) speech. 


\section{Conclusions}

In view of the above results the following conclusions can be drawn:

- the subjective overall evaluation of loudspeaker systems takes place in a one-dimensional space

- changes in the position (angle of observation) of loudspeaker systems are perceived on the basis of the impression of sharpness

- differentiation between loudspeaker systems with ana$\log$ and digital crossover networks is based on the expression of clearness - in the case of music signals and loudness - in the case of speech signals

\section{References}

[1] M. Niewiarowicz, P. Pekkala, in: Conference of the State Committee for Scientific Researches, Kraków 1995, p. 31.

[2] M. Niewiarowicz, in: 15th International Congress on Acoustics, Trondheim 1995, p. 245.

[3] M. Niewiarowicz, in: Proc. 98th AES Convention, Paris 1995, preprint 4004.
[4] M. Niewiarowicz, H. Łopacz, Archiv. Acoust. 33, 541 (2008).

[5] M. Niewiarowicz, H. Łopacz, in: Proc. XLV Open Seminar on Acoustics, Ed. T. Hornowski, Polish Acoustical Society, Poznań 1998, p. 457.

[6] M.O. Hafksford, J. Audio Eng. Soc. 95, 37 (1997).

[7] P. Dziechciński, in: VIII Symposium on the news in audio and video techniques, Warszawa 2002, p. 5.

[8] A. Ilyeni, P. Korpasi, Acustica 49, 334 (1981).

[9] F.E. Toole, J. Audio Eng. Soc. 33, 33 (1985).

[10] A. Furmann, Ph.D. Thesis, A. Mickiewicz University, Poznań 1991.

[11] W.J.M. Levelt, J.P. Geer, R. Plomp, Brit. J. Math. Statist. Psychol. 19, 163 (1966).

[12] A. Gabrielsson, Scand. J. Psychol. 20, 159 (1979).

[13] A. Furmann, E. Hojan, M. Niewiarowicz, P. Perz, J. Audio Eng. Soc. 38, 837 (1990).

[14] J.D. Carroll, J.J. Chang, Psychometrika 35, 283 (1970). 